\title{
Rate of Viral Re-Suppression and Retention To Care Among HIV Infected Patients on Second Line Antiretroviral Therapy at Dessie Comprehensive Specialized Hospital, Northeast Ethiopia.
}

Shambel Wedajo ( $\sim$ Swedajo@gmail.com )

Wollo University

\section{Getu Degu}

Bahir Dar University

\section{Amare Deribew}

Nutrition International ( $\mathrm{NI})$ in Ethiopia

Fentie Ambaw

Bahir Dar University

\section{Research Article}

Keywords: Second line therapy, Re-suppression, Retention to care, Attrition

Posted Date: February 16th, 2021

DOI: https://doi.org/10.21203/rs.3.rs-193273/v1

License: (c) (i) This work is licensed under a Creative Commons Attribution 4.0 International License. Read Full License 


\section{Abstract}

Background: In Ethiopia, first line antiretroviral therapy failure is growing rapidly. However, unlike first line therapy, to date, very little is known about the outcomes of second line therapy. Thus, this study assessed the rate of viral resuppression and retention to care and their predictors among HIV infected patients on second line therapy.

Methods: A retrospective cohort study was conducted among 642 HIV infected patients on second line therapy at Dessie Comprehensive Specialized Hospital, northeast Ethiopia from October 2016 to November 2019. Proportional Cox regression model was computed to explore predictors of viral re-suppression (Viral load less than 1000 copies $/ \mathrm{mL}$ ) and attrition to care.

Results: Out of 642 subjects, 19 (3\%), 44(6.9\%), 70(10.9\%), and 509(79.3\%) patients were lost to follow up, died, transferred out, and alive on care, respectively. Similarly, $82.39 \%$ (79.24 - 85.16\%) patients had achieved viral resuppression, with 96 per 100-person year rate of re-suppression in 550.16 year follow up. Patients who switched timely to second line therapy were at a high rate of viral re-suppression than patients who delayed to switch [Adjusted Hazard Rate, AHR = 1.43 (95\%Cl: 1.17-1.74)]. Not having drug substitution history $[\mathrm{AHR}=1.25$ (95\% Cl: $1.02-$ 1.52)] was positively associated with viral re-suppression, in contrast, being on anti-TB treatment [AHR $=0.67(95 \%$ Cl: $0.49-0.91)$ ] negatively associated with viral re-suppression.

By excluding transferred out cases, 63 (11\%, 95\% Cl: $9.6-13.8 \%)$ out of 572 patients were failed to retain on care with 7.1 per 100 person year rate of attrition in 887.25 year observation. Patients who were ambulatory or bedridden at the time of therapy switch were more at risk of attrition to care as compared with workable patients [Adjusted Hazard Rate, AHR= 2.61 (95\% Cl: 1.40-4.87]. Similarly, being not virally re-suppressed [AHR= 6.87 (95\% Cl: 3.8612.23)], $\mathrm{CD} 4$ count $<=450$ cells $/ \mathrm{mm}^{3}$ [AHR= $2.61(95 \% \mathrm{Cl}: 1.40-4.87]$ were also positively associated with attrition to care.

Conclusions: A significant number of patients had failed to achieve viral re-suppression and to retain on care. Most identified factors related to patient monitoring. Hence, patient centered intervention should be strengthen, besides treatment switch.

\section{Background}

According to UNAIDS 2019 report, globally 38 million people are living with HIV, and Africa accounts 25.9 million, of which, eastern and southern Africa takes 20.7 million (54.5 \%) (1). According to Ethiopian Public Health Institute (EPHI) HIV estimation, 737,186 people living with HIV are estimated in 2019 (2). Globally and in Ethiopia $66.8 \%$ and $60 \%$ of people living with HIV accessed antiretroviral therapy in the year of 2019 , respectively $(1,3)$.

The introduction of antiretroviral therapy (ART) significantly reduces HIV related morbidity and mortality. Nevertheless, the proportion of first line regimen failure is growing fast along with the overall patient number and duration on ART. A mathematical model done in sub-Saharan Africa to estimate the need of second-line antiretroviral therapy showed that in 2020 and 2030, 2.9\%-15.6\% and 6.6\%-19.6\% PLHIV will receive second-line ART, respectively(4). A systematic review done in Ethiopia also showed that 15.9\% (11.6-20.1\%) People Living with HIV(PLHIV) had experienced first line regimen failure that requires switching to second-line therapy(5). Second line therapy requires more than double cost of the first line therapy(6) as well as it is the last treatment option in most low-income counties. 
According to DHIS2 2018 report, 3.2\% out of total clients active on ART were taking second line antiretroviral regimen in Ethiopia. However, unlike first line therapy, to date, very little is known about the outcomes of second line therapy; viral re-suppression, retention to care, death, loss to follow up, and their predictors. Treatment failure patients expected to achieve viral re-suppression; having viral load measurement below $1000 \mathrm{copies} / \mathrm{ml}$, and improve clinical condition after the commencement of second line therapy. However, this may not be true for all patients. Factors for poor treatment outcome may relate with clinical and non-clinical conditions as well as vary from place to place. Hence, it is better to explore why certain patients failed to achieve viral re-suppression and attrite from care.

A better understanding of the outcomes of second line therapy allows policy makers and implementer to design interventions. Consequently, prolong the use and effectiveness of second line therapy. Besides, it reduce the risk of further treatment failure that limit the switch to more expensive third-line regimen. Hence, this study was conducted to determine the rate of viral re-suppression and retention to care and their predictors among HIV infected individuals on second line antiretroviral therapy.

\section{Methods}

\section{Study design and setting}

A retrospective cohort study was conducted on HIV infected patients who started second line antiretroviral therapy at Dessie Comprehensive Specialized Hospital (DCSH) from October 2016 to November 2019. Dessie Comprehensive Specialized Hospital is located in the Amhara region, northeast Ethiopia that serves the top HIV burden area in the nation (7). Currently 5557adult patients are taking first line antiretroviral therapy and 1076 were ever enrolled to second line therapy.

During three-year follow up period, 686 adult patients started second line antiretroviral therapy. By excluding patients who had taken second line antiretroviral for less than six months or had no viral load measurement, the remaining 642 patients on second line therapy were included to this study.

In Ethiopia, current standard first line antiretroviral therapy consists of a combination of two Nucleoside Reverse Transcriptase Inhibitors (NRTIs) as a backbone; Lamivudine (3TC) and Abacavir (ABC), or Zidovudine (ZDV) or Tenofovir (TDF) and Dolutegravir (DTG) as Integrase strand transfer Inhibitors (INSTIs) or Efavirenz (EFV) as NonNucleoside Reverse Transcriptase Inhibitors (NNRTIs) as a base.

A second line regimen is a combination of three ARV drugs (at least two of which are new to the patient); two Nucleoside Reverse Transcriptase Inhibitors (NRTIs) and one protease Inhibitors (PIs); Lopinavir/ ritonavir (LPV/r) or Atazanavir / ritonavir $(A T V / r)(8,9)$. Treatment success is monitored using viral load $(V L)$ measurement in which viral load measurement is done after initiation of ART at 6 months, 12 months and every 12 months. If two consecutive VL results more than 1000 copies/ mL with enhanced adherence support confirm failure of the current treatment regimen and patients will switch to second line regimen. In Ethiopia, viral load measurement was started in 2016 on top 20 burden areas including Dessie comprehensive specialized hospital $(10,11)$.

\section{Outcome variable and predictors}

The primary outcome variable in this study was viral re-suppression (event) which is defined as having viral load measurement below 1000 copies/mL after six-months exposure of second antiretroviral therapy, unless considered as virological failure $(8,9)$. Retention to care is defined as alive and in care on a second - line regimen at the time of 
data collection. Died or Loss to follow up (missed contact with the health facility for three consecutive months) patients are considered as attrition to care.

Socio-demographic profiles: age, sex, marital status, educational status and disclosure status.

Clinical profile at therapy switch includes: year on ART, body mass index, functional status, WHO clinical stage, TB treatment status, TB preventive therapy (INH), CD4 cell/mm³ , Viral load, first line ART regimen before switch, drug substitution history during first line therapy, $2^{\text {nd }}$ line ARV regimen, medication adherence, and time between first virological failure and initiation of second line therapy. Timely switch is considered if the patient starts second line treatment at third month after the first virological failure, otherwise it is considered as delayed to switch $(8,9)$.

\section{Data collection procedures}

The data were collected using a structured data extraction checklist by reviewing chronic HIV follow up form, ART registration book, and SMART care electronic database. The extraction sheet was prepared using notational consolidated antiretroviral guideline (9). Document review was done for patients who start second line antiretroviral therapy from October 2016 to November 2019. Documents were retrieved using patient Medical Record Number (MRN) and ART registration number. The qualities of data were secured by triangulation of data sources; minimize data incompleteness and inconsistency, using a pretested questionnaire, employing trained data collectors, and conducting on-site supervision.

\section{Statistical analysis}

Data were cleaned and entered into EpiData Version 3.1 software, and then, exported to Stata version 14 for further analysis. Proportion for categorical variables and median with inter-quartile ranges (IQR) for continuous variables were computed after considering distributional assumption tests. Incidence rate of viral re-suppression and attrition to care was calculated using person time of observations. Person-time is the sum of the number of years contributed by study participants in the follow-up period. Proportional Cox regression model was computed to explore significant predictor variables. Proportional hazard ratio assumption was checked using global goodness of fit (Schoenfeld residuals) test. In multivariable Cox model, P-value $<0.05$ was considered statistically significant and presented with $95 \% \mathrm{Cl}$.

\section{Results}

\section{Description of study population}

During three years follow up period, 19 (3\%), 44(6.9\%), 70(10.9\%), and 509(79.30\%) of 642 patients were lost to follow up, died, transferred out and alive on care, respectively. Regarding treatment success, 529(82.39\%) of 642 patients had achieved viral re-suppression $(\mathrm{VL}<1000$ copies $/ \mathrm{mL})$ at the end of follow up period.

Out of 642 participants, 359(55.9\%) were female and nearly fifty percent were married. One third of participants had not attended formal education, and 181(28.2\%) were unemployed. Concerning disclosure of HIV status, 538(83.8\%) patients disclosed their status for at least one of the family members. Table 1: Socio-demographic profile of HIV infected patients on second line antiretroviral therapy at Dessie Comprehensive Specialized Hospital, northeast Ethiopia, October 2016 - November 2019. ( $n=642)$.

\section{Clinical profiles of HIV infected patients at the start of second line therapy}


Out of 642 participants, $466(72.8 \%)$ and 140(21.8\%) participants had BMI > $=18.5 \mathrm{~kg} / \mathrm{m}^{2}$ and CD4 count greater than 450 cells $/ \mathrm{mm}^{3}$ at the start of second line therapy. Similarly, 85(13.2\%) and 19(3\%) participants were at advanced clinical stage and bedridden at therapy switch. Fifteen percent of participants were on anti-TB treatment during the first six months of second line therapy and 350(54.51\%) had not taken TB preventive therapy (INH). TDF3TC-EFV 219(34.1\%) and TDF-3TC-ATV/r (283(44.1\%) were the most prescribed first and second line antiretroviral regimens.

Regarding medication adherence and drug substitution, 574(89.4\%) patients had good adherence while on second line antiretroviral therapy and 448(69.8\%) patients had not drug substitution history while on first line therapy. The median time between the first virological failure and the start of second line therapy was 5(IQR 3-8) month and $431(67.13 \%)$ participants delayed to switch timely after fist virological failure. Table 2: Clinical profiles of HIV infected patients at the start of second line therapy, Dessie Comprehensive Specialized Hospital, northeast Ethiopia, October 2016 - November 2019. $(n=642)$.

\section{Rate of viral re-suppression and predictors}

Out of 642 patients who started second line therapy, 82.39\% (95\% Cl: $79.24-85.16 \%)$ had achieved viral resuppression, with 96 per 100-person year rate of re-suppression in 550.16-year follow up. After six months exposure of second line therapy, 209 patients had still high viral load measurement and passed to enhanced adherence support. From which, 96(46\%) patients had re-suppressed, 74(35.4\%) ongoing viremia and 39 were confirmed treatment failure. Table 3: Life table on viral re-suppression among HIV infected patients on second line therapy, Dessie Comprehensive Specialized Hospital, from October 2016 - November 2019. $(n=642)$.

Patients who switched timely to second line antiretroviral therapy were 1.43 time more likely to have viral resuppression at any time as compared to delayed patients, while holding all other variables in the model constant [AHR=1.43, (95\% Cl: 1.17-1.74)]. Similarly, patients who had no first line antiretroviral drug substitution history were 1.25 times more likely to have viral re-suppression at any time as compared with drug substituted patients [AHR=1.25, (95\% Cl: 1.02-1.52)].

Patients who had viral load measurement 1000 - 4140 copies $/ \mathrm{mL}$ and 4141 - 13195 copies/mL at therapy switch were 1.60 and 1.38 times more likely to have viral re-suppression at any time as compared with the reference category $(\mathrm{VL}>=52,753$ copies $/ \mathrm{mL})$, respectively. Patients who were on anti-TB treatment during the first six months of second line therapy were on average 33\% decrease on the likely of viral re-suppression as compared with the counter parts $[\mathrm{AHR}=0.67,(95 \% \mathrm{Cl}: 0.49-0.91)]$, while holding all other variables in the model constant. Table 4: Bivariable and multivariable proportional Cox regression model on determinants of viral re-suppression among HIV infected patients on second line therapy, Dessie Comprehensive Specialized Hospital, October 2016 - November 2019. $(n=642)$.

\section{Attrition to care and predictors}

By excluding transferred out cases, $63(11 \%, 95 \% \mathrm{Cl}: 9.6-13.8 \%)$ out of 572 patients were failed to retain on care with 7.1 per 100 person year rate of attrition in 887.25 year observation. From attrition patients, 19(3.3\%) and $44(7.7 \%)$ were lost to follow up and died, respectively. The cumulative proportions of attrition to care at year 1, 2, 3 were $7.16 \%$ (95\% Cl: 5.2-9.8\%), 13.36\%(95\% Cl: 10.31-17.23\%), $21.62 \%(95 \% \mathrm{Cl}: 16.30-28.35 \%)$, respectively.

Patients who were ambulatory or bedridden at the time of therapy switch were 2.61 times more at risk of attrition to care at any time due to death or loss to follow up as compared with workable patients [AHR=2.61, (95\% Cl: 1.40 - 
4.87)]. Similarly, patients whose CD4 count less than 450 copies $/ \mathrm{mm}^{3}$ were 3.81 times more at risk of attrition to care at any time as compared with the counter parts [AHR=3.81, (95\% Cl: $1.17-12.39)$ ]. Patients who failed to achieve viral re-suppression were 6.87 times more at risk of attrition to care as compared with viral re-suppressed patients[AHR= 6.87, (95\% Cl: 3.86-12.23)]. Table 5: Bivariable and multivariable proportional Cox regression model on determinants of attrition to care among HIV infected patients on second line antiretroviral therapy, Dessie Comprehensive Specialized Hospital, northeast Ethiopia, October 2016 - November 2019. (n=572).

\section{Discussion}

The present study shows that one in every five patients on second line therapy failed to achieve viral re-suppression. This finding was in agreement with studies conducted in resource limited settings (12-17) and higher than a study conducted in south Africa(18). This variation is due to a difference in viral load measurement classification. Viral load measurement below 400 copies/mL and 1000 copies/mL was taken as cutoff point to define viral resuppression in study conducted in South Africa and current study, respectively. In general, a significant number of patients on the second line therapy had not achieved viral re-suppression (virological failure). This has both clinical and public health implications. Clinically, it increases the risk of drug resistance, second line treatment failure, and demand of high cost third line antiretroviral therapy. Besides, at the public level, it also increases the chance of HIV transmission, even resistant strain and far remote to achieve UNAIDS settled targets on viral suppression which says $95 \%$ of people on treatment will have suppressed viral load(19).

Eleven percent of patients on second line therapy had experienced attrition to care. This finding was in line with a study done in Rwanda(14) and higher than the result of other studies $(15,16,18,20,21)$. This variation is due to a difference in computing attrition, that is transferred out cases were included in previous studies but not in this study. Non retained patients have a greater risk of morbidity, mortality as well as increase the rate of HIV transmission and health care costs. Non retained HIV patients had an estimated rate of 6.6 transmissions per 100 person-years, compared with individuals engaged in the care (22).

Drug substitution history is negatively associated with viral re-suppression. Frequent first line antiretroviral drug substitution leads to reduction of subsequent treatment options and enforces reuse of previously substituted drugs. Consequently, there will be an increase the chance of drug resistant and fail to re-suppress the viral load. In second line antiretroviral therapy, NRTI backbone drug substituted in the same class drug, which may be previously used $(8,9)$. Hence, appropriate drug selection at the commencement of first line antiretroviral therapy had a vital role on the effectiveness of subsequent therapy.

Similarly, anti-TB treatment and viral re-suppression were also inversely associated, which is consistent with a study conducted in South Africa (23). This association may be explained by the occurrence of infection (tuberculosis) which will flare-up the viral replication. Furthermore, there may be an interaction of anti-TB treatment drugs and second line antiretroviral therapy; rifampicin a potent cytochrome P450 3A4 liver enzyme inducer, which significantly reduces the serum levels of the PI drugs and consequently it reduce the rate of viral re-suppression. According to WHO - 2016 and national consolidate ART guidelines, for TB-HIV Co-infected patients the dose of lopinavir/ritonavir (LPV/r) should be adjusted; doubling the daily dose (i.e. LPV/r $800 \mathrm{mg} / 200 \mathrm{mg}$ twice daily) or a super-boosted dose of RTV (i.e. LPV/r 400 mg/400 mg twice daily)(8,9). However, this may also leads to drug intolerance and poor medication adherence. In line with this, a cohort study done in South Africa on adverse drug (ADR) reaction revealed that patients with poor health at time of switch were at a high risk of ADR when receiving second lien therapy(24). 
Having high viral load at the commencement of second line antiretroviral therapy is inversely related to viral resuppression. This finding is also supported by many studies(14,16,17,25-28). Hence, switching to second line therapy may not be a warranty for viral re-suppression for patients with high viral set point. It requires further close monitoring and strengthening of the implementation of enhanced adherence counseling for such type clients.

Delayed regimen switch after the first virological failure is associated with poor treatment outcomes. This finding was consistent with a prospective study conducted in South Africa(18). According to WHO-2016 and national consolidated ART guidelines, a patient should be switched to next level therapy when having two consecutive viral load measurement greater than 1000 copies/mL in three months apart, with enhanced adherence Support following the first viral load test $(8,9)$. Failure to switch timely (i.e. at three months after EAC) will give sufficient time for viral replication, development of resistance strain, which result in poor treatment outcomes. Studies done in Africa showed that delayed between first virological failure and commencement of second line therapy significantly related to poor immunological and virological out comes as well as increased opportunity infection and mortality(29-31). Delay in therapy switch may related to an individual, health professional or health facility factors, which may require further investigation.

Being ambulatory, bedridden and lower CD4 cell count at therapy switch had an increased risk of lost to follow up and death. This finding was consistent with the studies done in Ethiopia on patients on first line therapy $(14,15,17,27,32)$. Hence, accessing routine viral load monitoring for early diagnosis of treatment failure will increase the probability of patient retention on care, instead of waiting until immunological failure or clinical advancement. This association may be explained by poor immunological and clinical condition can easily leads to drug intolerance as well as poor drug compliance and this may further contribute poor treatment out comes and failed to retained on care.

Similarly, patients with virological failure while on second-line treatment were also an increases risk of attrition, which is supported by a retrospective cohort study done in south Africa(15).Virally suppressed patients would have a stable clinical condition, feel self-control, and had better perceived quality of life that prolongs the chance of staying on care. This result implies that conducting routine viral load monitoring had a key role in program evaluation besides showing the success of treatment. Hence striving to achieve the $3^{\text {rd }} 95$ is one means of reducing patient attrition.

\section{Strength and limitation of the study}

This study has several strengths. First, the study is the first of its kind in Ethiopia to provide first-hand information on the subject to improve the outcomes of patients on second line therapy. Second, it was conducted on large sample size as well as used different data sources (Chronic HIV follow up form, ART registration book, and SMART care electronic database) that increases consistency as well as minimize incompleteness. In contrast, this study is not free from limitations. It failed to collect and analyze data on behavioral, social and psychological factors as well as facility level determinants of viral re-suppression and attrition from care. Further, this study was unable to consider other clinical profiles like comorbidities and organ function results. All the above limitations were due the nature of study design. Therefore, confounding through the unmeasured covariates need to be considered while interpreting the reported associations.

\section{Conclusion}


A significant number of patients had failed to achieve viral re-suppression and to retain on care after enrollment to second line antiretroviral program. Most identified factors related to patient monitoring and clinical profiles. Hence, patient center interventions should be crafted and implanted on the identified predictors.

\section{Abbreviations}

ART: antiretroviral therapy, PLHIV: People Living with HIV, VL: viral load, NRTIs: Nucleoside Reverse Transcriptase Inhibitors (NRTIs), NNRTIs: Non-Nucleoside Reverse Transcriptase Inhibitors (NNRTIs), INSTIs: Integrase strand transfer Inhibitors, PIs: Protease Inhibitors, DTG: Dolutegravir 3TC: Lamivudine, ABC: Abacavir, AZT: Zidovudine, TDF: Tenofovir, EFV: Efavirenz, NVP: Nevirapin, LPV/r: Lopinavir/ ritonavir, ATV/r: Atazanavir/ritonavir, BMI: Body Mass Index, IQR: Interquartile range, ADR: adverse drug reaction, Isoniazid preventive therapy (INH), Medical Record Number (MRI), DHIS2: District health information system, AHR: adjusted hazard ratio.

\section{Declarations}

\section{Ethics approval and consent to participate}

This study was conducted in accordance with the declaration of Helsinki. Ethical approval was secured from Institutional Review Board/IRB/ of Bahir Dar University, College of Medicine and Health Science with reference number 00224/2020. Further, authorization was obtained from Dessie Comprehensive Specialized Hospital in order to use of anonymized data. Besides, the confidentiality of obtained information assurance made by using code numbers rather than personal identifiers and by keeping the checklist locked. Since we used secondary sources, informed consent was waived that approved by Institutional Review Board/IRB/ of Bahir Dar University, College of Medicine and Health Science.

\section{Availability of data and materials}

The datasets used and/or analyzed during the current study are available from the corresponding author on reasonable request.

\section{Consent for publication}

Not applicable.

\section{Competing interests}

None of the authors has any conflict of interest.

\section{Funding}

This research founded by Bahir Dar University College of Medicine and Health science, School of Public Health.

\section{Authors' contributions}

SW contributed towards conception, design, data collection, interpretation of data and drafting the manuscript. GD, $A D$ and FA contributed towards conception, design, interpretation of data and drafting the manuscript. All authors read and approved the final manuscript. 
We acknowledge the funding support provided by Bahir Dar University College of Medicine and Health science, School of Public Health as well as Dessie comprehensive hospital for giving full background data.

\section{Author details}

${ }^{1}$ School of Public Health, CMHS, Wollo University, ${ }^{2}$ School of Public Health, CMHS, Bahir Dar University, ${ }^{3}$ Country Director, Nutrition International (NI) in Ethiopia

\section{References}

1. Communications and Global Advocacy UNAIDS. UNAIDS FACT SHEET. Global HIV Statistics. Ending the AIDS epidemic. 2020.

2. The Ethiopian Public Health Institute. HIV Related Estimates and Projections for Ethiopia-2017 [Internet]. 2017. Available from:

https://www.ephi.gov.et/images/pictures/download2009/HIV_estimation_and_projection_for_Ethiopia_2017.pdf

3. PEPFR. Ethiopia Country Operational Plan Strategic Direction Summary. 2020.

4. Estill J, Ford N, Salazar-vizcaya L, Haas AD, Blaser N, Habiyambere V, et al. The need for second-line antiretroviral therapy in adults in sub-Saharan Africa up to 2030 : a mathematical modelling study. Lancet HIV [Internet]. 2016;3018(16):1-8. Available from: http://dx.doi.org/10.1016/S2352-3018(16)00016-3

5. Elvstam O, Medstrand P, Yilmaz A, Isberg PE, Gisslen M, Bjorkman P. Virological failure and all-cause mortality in HIV-positive adults with low-level viremia during antiretroviral treatment. PLoS One. 2017;12(7):1-15.

6. WHO. Ethiopia HIV Country Profile: 2016 WHO/HIV/2017. 2017.

7. Worku ED, Asemahagn MA, Endalifer ML. Epidemiology of hiv infection in the amhara region of ethiopia, 2015 to 2018 surveillance data analysis. HIV/AIDS - Res Palliat Care. 2020;12:307-14.

8. WHO. The use of antiretroviral drugs for treating and preventing hiv infection. [Internet]. 2016. 99-152, 402 p. Available from: https://apps.who.int/iris/bitstream/handle/10665/208825/9789241549684_eng.pdf? sequence $=1 \&$ isAllowed $=y$

9. Ethiopia Federal Ministry of Health. NATIONAL CONSOLIDATED GUIDELINES FOR COMPREHENSIVE HIV PREVENTION, CARE AND Treatment. 2018. 1-238 p.

10. EPHI. Ethiopian Public Health Institution National HIV Reference Laboratory. 2016.

11. Medicne A societ laboratory. National Review on HIV Viral Load Testing and Early Infant Diagnosis in Ethiopia [Internet]. 2020. Available from: https://aslm.org/news-article/national-review-meeting-hiv-viral-load-testingearly-infant-diagnosis-ethiopia/

12. Therapy SA, Fox MP. Predicting the Need for Third-Line Antiretroviral Therapy by Identifying Patients at High Risk for Failing. Clin Epidemiol Res. 2017;31(5):205-12.

13. Collier D, Iwuji C, Derache A, Oliveira T De, Okesola N, Calmy A, et al. Virological Outcomes of Second-line Protease Inhibitor - Based Treatment for Human Immunodeficiency Virus Type 1 in a High-Prevalence Rural South African Setting: A Competing-Risks Prospective Cohort Analysis. Clin Infect Dis MAJOR Artic Virol. 2017; $(\mathrm{Xx} \mathrm{Xxxx):1-11.}$

14. Nsanzimana S, Semakula M, Ndahindwa V, Remera E, Sebuhoro D, Uwizihiwe JP, et al. Retention in care and virological failure among adult HIV+ patients on second-line ART in Rwanda: a national representative study. BMC Infect Dis. 2019;19(1):1-9. 
15. Gumede SB, Fischer A, Venter WDF, Lalla-Edward ST. Descriptive analysis of World Health Organizationrecommended second-line antiretroviral treatment: A retrospective cohort data analysis. South African Med J. 2019;109(12):919-26.

16. Chakravarty J, Sundar S, Chourasia A, Singh PN, Kurle S, Tripathy SP, et al. Outcome of patients on second line antiretroviral therapy under programmatic condition in India. 2015;

17. Shearer K, Evans D, Moyo F, Rohr JK, Berhanu R, Van Den Berg L, et al. Treatment outcomes of over 1000 patients on second-line, protease inhibitor-based antiretroviral therapy from four public-sector HIV treatment facilities across Johannesburg, South Africa. Trop Med Int Heal. 2017;22(2):221-31.

18. Fox MP, Ive P, Long L, Maskew M, Sanne I. High Rates of Survival , Immune Reconstitution , and Virologic Suppression on Second-Line Antiretroviral Therapy in South Africa. Epidemilogoy Soc Sci. 2010;53(4):500-6.

19. UNAIDS. 90-90-90 An ambitious treatment target to help end the AIDS epidemic [Internet]. 2014.40 p. Available from: http://www.unaids.org/sites/default/files/media_asset/90-90-90_en.pdf

20. Mwasakifwa GE, Moore C, Carey D, Amin J, Penteado P, Losso M, et al. Relationship between untimed plasma lopinavir concentrations and virological outcome on second-line antiretroviral therapy. Aids. 2018;32(3):357-61.

21. Thu N, Kyaw T, Kumar AM V, Oo MM, Oo HN, Kyaw WY, et al. Long-term outcomes of second-line antiretroviral treatment in an adult and adolescent cohort in Myanmar. Glob Health Action [Internet]. 2017;10(1). Available from: https://doi.org/10.1080/16549716.2017.1290916

22. Li Z, Purcell DW, Sansom SL, Hayes D, Hall HI. Vital Signs: HIV Transmission Along the Continuum of Care United States , 2016. 2019;68(11):267-72.

23. Collier D, Iwuji C, Derache A, De Oliveira T, Okesola N, Calmy A, et al. Virological outcomes of second-line protease inhibitor-based treatment for human immunodeficiency virus type 1 in a high-prevalence Rural South African setting: A competing-risks prospective cohort analysis. Clin Infect Dis. 2017;64(8):1006-16.

24. Onoya D, Hirasen K, Berg L Van Den, Miot J, Long LC. Adverse Drug Reactions Among Patients Initiating Second - Line Antiretroviral Therapy in South Africa. Drug Saf [Internet]. 2018;41(12):1343-53. Available from: https://doi.org/10.1007/s40264-018-0698-3

25. Mocroft A, Phillips AN, Miller V, Gatell J, Van Lunzen J, Parkin JM, et al. The use of and response to second-line protease inhibitor regimens: Results from the EuroSIDA study. Aids. 2001;15(2):201-9.

26. Desai M, Dikshit R, Patel D, Shah A. Early outcome of second line antiretroviral therapy in treatment-experienced human immunodeficiency virus positive patients. Perspect Clin Res. 2013;4(4):215.

27. Häggblom A, Santacatterina M, Neogi U, Gisslen M, Hejdeman B, Flamholc L, et al. Effect of therapy switch on time to second-line antiretroviral treatment failure in HIV-infected patients. PLoS One. 2017;12(7):1-14.

28. Thao VP, Quang VM, Wolbers M, Anh ND, Shikuma C, Farrar J, et al. Second-line HIV therapy outcomes and determinants of mortality at the largest HIV referral center in Southern Vietnam. Med (United States). 2015;94(43).

29. Ssempijja V, Nakigozi G, Chang L, Gray R, Wawer M, Ndyanabo A, et al. Rates of switching to second-line antiretroviral therapy and impact of delayed switching on immunologic, virologic, and mortality outcomes among HIV-infected adults with virologic failure in. BMC Infect Dis. 2017;1-10.

30. Murphy RA, Court R, Maartens G. Second-Line Antiretroviral Therapy in Sub-Saharan Africa: AIDS Res Hum petroviruses. 2017;33(12):1181-4.

31. Maya L. Petersena, Linh Trana, Elvin H. Gengb, Steven J. Reynoldsc, d, e, Andrew Kambuguf, Robin Woodg, David R. Bangsbergh, i, Constantin T. Yiannoutsosj, Steven G. Deeksb and JNM. Delayed switch of antiretroviral 
therapy after virologic failure associated with elevated mortality among HIV-infected adults in Africa. NIH Public Access. 2015;28(14):2097-107.

32. Moges NA, Olubukola A, Micheal O, Berhane Y. HIV patients retention and attrition in care and their determinants in Ethiopia: a systematic review and meta-analysis. BMC Infect Dis. 2020;20(439):1-24.

\section{Tables}

Table 1: Socio-demographic profile of HIV infected patients on second line antiretroviral therapy at Dessie Comprehensive Specialized Hospital, northeast Ethiopia, October 2016 - November 2019. (n=642). 


\begin{tabular}{|c|c|c|c|}
\hline Characteristics & $\mathrm{n}(\%)$ & Viral re-suppressed & Censored \\
\hline \multicolumn{4}{|l|}{ Sex } \\
\hline Female & $359(55.9)$ & $302(84.12)$ & $57(15.88)$ \\
\hline Male & $283(44.1)$ & $227(80.21)$ & $56(19.79)$ \\
\hline \multicolumn{4}{|l|}{ Marital status } \\
\hline Single & 199(31.0) & $152(76.38)$ & $47(23.62)$ \\
\hline Married & 298(46.4) & 266(89.26) & $32(10.74)$ \\
\hline Divorce & $83(12.9)$ & 61(73.49) & $22(26.51)$ \\
\hline Widowed & $62(9.7)$ & $50(80.65)$ & $12(19.35)$ \\
\hline \multicolumn{4}{|l|}{ Religion } \\
\hline Orthodox & $282(43.9)$ & $240(85.11)$ & $42(14.89)$ \\
\hline Muslim & $325(50.6)$ & $260(80)$ & $65(20)$ \\
\hline Protestant & $23(3.6)$ & $19(82.61)$ & $4(17.39)$ \\
\hline Catholic & $12(1.9)$ & $10(83.33)$ & $2(15.67)$ \\
\hline \multicolumn{4}{|l|}{ Educational status } \\
\hline No education & $216(33.6)$ & 175(81.02) & $41(18.89)$ \\
\hline Primary & 182(28.3) & 156(85.71) & $26(14.29)$ \\
\hline Secondary & 157(24.5) & 123(78.34) & $34(21.66)$ \\
\hline Tertiary & $87(13.6)$ & $75(86.21)$ & $12(13.79)$ \\
\hline \multicolumn{4}{|l|}{ Occupational status } \\
\hline Government employee & $65(10.1)$ & $57(87.69)$ & $8(12.31)$ \\
\hline House wife & $145(22.6)$ & $125(86.21)$ & $20(13.79)$ \\
\hline Merchant & $103(16)$ & $77(74.76)$ & $26(25.24)$ \\
\hline Farmer & 114(17.8) & $99(86.84)$ & 15(13.19) \\
\hline Unemployed & 181(28.2) & 141(77.9) & $40(22.10)$ \\
\hline Private employee & $34(5.3)$ & $30(88.24)$ & $4(11.79)$ \\
\hline \multicolumn{4}{|l|}{ Disclosure status } \\
\hline Disclosed & $538(83.8)$ & $450(83.64)$ & $88(16.36)$ \\
\hline Not disclosed & 104(16.2) & $79(75.96)$ & $25(24.04)$ \\
\hline Duration on first line antiretroviral therapy, median (IQR) (year) & $8(5.4-10.1)$ & & \\
\hline Age, median (IQR)(year) & $35(27-42)$ & & \\
\hline
\end{tabular}

Table 2: Clinical profiles of HIV infected patients at the start of second line therapy, Dessie Comprehensive 
Specialized Hospital, northeast Ethiopia, October 2016 - November 2019.(n=642).

Page $13 / 16$ 


\begin{tabular}{|c|c|c|c|}
\hline Clinical profiles & $n(\%)$ & $\begin{array}{l}\text { Viral re- } \\
\text { suppression }\end{array}$ & Censored \\
\hline \multicolumn{4}{|l|}{ BMI } \\
\hline$>=18.5 \mathrm{~kg} / \mathrm{m}^{2}$ & $466(72.8)$ & $400(85.84)$ & $66(14.16)$ \\
\hline$<18.5 \mathrm{~kg} / \mathrm{m}^{2}$ & $176(27.2)$ & $129(73.30)$ & $47(26.70)$ \\
\hline \multicolumn{4}{|l|}{ Functional status } \\
\hline Workable & $542(84.4)$ & $463(85.42)$ & $79(14.58)$ \\
\hline Ambulatory & $81(12.6)$ & $55(67.90)$ & $26(32.10)$ \\
\hline Bedridden & 19(3.0) & 11(57.89) & $8(42.11)$ \\
\hline \multicolumn{4}{|l|}{ WHO clinical stage } \\
\hline | and || & $557(86.8)$ & $475(85.25)$ & $82(14.72)$ \\
\hline $\mathrm{III}$ and IV & $85(13.2)$ & $54(63.53)$ & $31(36.47)$ \\
\hline \multicolumn{4}{|c|}{$\begin{array}{l}\text { TB treatment status at regimen switch or during the first six months } \\
\text { of second line therapy: }\end{array}$} \\
\hline \multicolumn{4}{|l|}{ On anti-TB treatment } \\
\hline \multirow[t]{2}{*}{ Not on anti-TB treatment } & $93(14.5)$ & $02(00.91)$ & $41(44.09)$ \\
\hline & $549(85.5)$ & 477(86.89) & 72(13.11) \\
\hline \multicolumn{4}{|l|}{ TB preventive therapy(INH) } \\
\hline Had not taken & $350(54.51)$ & $279(79.71)$ & $71(20.29)$ \\
\hline Had taken & 292(45.49) & $250(85.62)$ & $42(14.38)$ \\
\hline \multicolumn{4}{|l|}{ CD4 cell/Ml } \\
\hline$<=450$ cell $/ \mathrm{mm}^{3}$ & $502(78.19)$ & 402(80.08) & $100(19.92)$ \\
\hline$>450 \mathrm{cell} / \mathrm{mm}^{3}$ & $140(21.81)$ & 127(90.71) & 13(9.29) \\
\hline \multicolumn{4}{|l|}{ First line ARV regimen } \\
\hline AZT-3TC-NVP & 195(30.4) & $158(81.03)$ & $37(18.97)$ \\
\hline AZT-3TC-EFV & $144(22.4)$ & 124(86.11) & 20(13.89) \\
\hline TDF-3TC-EFV & 219(34.1) & 176(80.37) & $43(19.63)$ \\
\hline TDF-3TC-NVP & $84(13.1)$ & $71(84.52)$ & $13(15.48)$ \\
\hline \multicolumn{4}{|c|}{ First line drug substitution history } \\
\hline Yes & 194(30.2) & $148(7629)$ & $46(23.71)$ \\
\hline \multirow[t]{2}{*}{ No } & $448(69.8)$ & $140(70.28)$ & $67(14.96)$ \\
\hline & & $381(85.04)$ & \\
\hline \multicolumn{4}{|l|}{ Second line ARV regimen } \\
\hline AZT-3TC-LPV/r & $17(2.6)$ & 15(88.24) & $2(11.76)$ \\
\hline
\end{tabular}

Page 14/ 16 


\begin{tabular}{|c|c|c|c|}
\hline AZT-3TC-ATV/r & 238(37.1) & 194(81.51) & $44(18.49)$ \\
\hline TDF-3TC-ATV/r & 21(3.3) & 18(85.71) & $3(14.29)$ \\
\hline TDF-3TC-ATV/r & 283(44.1) & 238(84.10) & $45(15.90)$ \\
\hline ABC-3TC-LPV/r & $13(2.0)$ & $8(61.54)$ & $5(38.46)$ \\
\hline ABC-3TC-ATV/r & 70(10.9) & $56(80.00)$ & $14(20.00)$ \\
\hline \multicolumn{4}{|l|}{ Medication adherence } \\
\hline Good adherence (> = 95\%) & $574(89.4)$ & 498(86.76) & 76(13.24) \\
\hline Poor adherence (<95\%) & $68(10.6)$ & $31(45.59)$ & $37(54.41)$ \\
\hline \multicolumn{4}{|c|}{$\begin{array}{l}\text { Time between first virological failure and initiation PIs therapy. } \\
\text { median (IQR) (month) }\end{array}$} \\
\hline Delayed to switch & $431(67.13)$ & $324(75.17)$ & 107(24.83) \\
\hline Timely switch & 211(32.87) & 205(97.16) & $6(2.84)$ \\
\hline Weight, median (IQR) (kg) & \multicolumn{3}{|l|}{$50(44-57)$} \\
\hline Viral load median (IQR) (copies/mL) & \multicolumn{3}{|c|}{ 13195(4140-52753) } \\
\hline
\end{tabular}

3TC: Lamivudine, ABC: Abacavir, AZT: Zidovudine, TDF: Tenofovir, EFV: Efavirenz , NVP: Nevirapin, LPV/r: Lopinavir/ ritonavir, ATV/r: Atazanavir / ritonavir , BMI: Body Mass Index, IQR: Interquartile range.

Table 3: Life table on viral re-suppression among HIV infected patients on second line therapy, Dessie Comprehensive Specialized Hospital, October 2016 - November 2019. ( $n=642)$

\begin{tabular}{|llllll|}
\hline $\begin{array}{l}\text { Year } \\
\text { time of failure }\end{array}$ & Number at start & $\begin{array}{l}\text { Re-suppression } \\
\text { (Event) }\end{array}$ & Censored & $\begin{array}{l}\text { Cumulative failure } \\
\text { (Re-suppression) }\end{array}$ & 95\% Cl \\
\hline 1 & 642 & 461 & 87 & 0.7703 & $0.7358-0.8031$ \\
\hline 2 & 94 & 63 & 20 & 0.9426 & $0.9167-0.9625$ \\
\hline 3 & 11 & 5 & 6 & 0.9785 & $0.9489-0.9929$ \\
\hline
\end{tabular}

Table 4: Bivariable and multivariable proportional Cox regression model on determinants of viral re-suppression among HIV infected patients on second line therapy, Dessie Comprehensive Specialized Hospital, October 2016 November 2019. $(n=642)$ 


\begin{tabular}{|c|c|c|c|c|}
\hline \multirow[t]{2}{*}{ Covariate } & \multicolumn{2}{|c|}{ Bi-variable Cox regression } & \multicolumn{2}{|l|}{$\begin{array}{l}\text { Multivariable Cox } \\
\text { regression }\end{array}$} \\
\hline & CHR(95\% Cl) P & P-value & AHR(95\% C) & P-value \\
\hline Age & $\begin{array}{l}1.09(1.003- \\
1.017)\end{array}$ & 0.080 & & \\
\hline Year on ART & $1.01(0.98-1.004)$ & 0.535 & & \\
\hline $\begin{array}{l}\text { Drug substitution history: had no substitution } \\
\text { history }\end{array}$ & $1.39(1.16-1.69)$ & 0.001 & $1.25(1.02-1.52)$ & 0.031 \\
\hline Time to switch: timely switched & $1.88(1.57-2.24)$ & $<0.001$ & $1.43(1.17-1.74)$ & $<0.001$ \\
\hline WHO clinical stage: Stage 3 or 4 & $\begin{array}{l}0.708(0.53- \\
0.94)\end{array}$ & 0.016 & & \\
\hline $\mathrm{CD} 4 \mathrm{cell} / \mathrm{mL}:>=450 \mathrm{cell} / \mathrm{mm}^{3}$ & $1.45(1.19-1.78)$ & $<0.001$ & & \\
\hline \multicolumn{5}{|l|}{ Viral Load (ref: 52756 - 702127) } \\
\hline $13196-52753$ copies $/ \mathrm{mL}$ & $1.29(1.01-2.68)$ & 0.049 & $1.14(0.88-1.50))$ & 0.302 \\
\hline 4141-13195 copies/mL & $1.68(1.31-2.16)$ & $<0.001$ & $1.38(1.07-1.80)$ & 0.015 \\
\hline 1000-4140 copies/mL) & $2.30(1.80-2.95)$ & $<0.001$ & $1.60(1.21-2.11)$ & 0.001 \\
\hline BMI: > = 18.5 kg/m² & $1.43(1.18-1.75)$ & $<0.001$ & & \\
\hline Being on anti - TB treatment & $0.52(0.39-0.69)$ & $<0.001$ & $\begin{array}{l}0.67(0.49- \\
0.91)\end{array}$ & 0.01 \\
\hline Medication Adherence : > = 95\% & $2.03(1.41-2.92)$ & $<0.001$ & & \\
\hline
\end{tabular}

Table 5: Bivariable and multivariable proportional Cox regression model on determinants of attrition to care among HIV infected patients on second line antiretroviral therapy, Dessie Comprehensive Specialized Hospital, northeast Ethiopia, October 2016 - November 2019. ( $n=572)$.

\begin{tabular}{|c|c|c|c|c|}
\hline \multirow{3}{*}{$\begin{array}{l}\text { Covariate } \\
\text { Educational status: Not formally educated }\end{array}$} & \multirow{2}{*}{\multicolumn{2}{|c|}{$\begin{array}{l}\text { Bi-variable } \\
\text { CHR(95\% Cl)P-value }\end{array}$}} & \multirow{2}{*}{\multicolumn{2}{|c|}{$\begin{array}{l}\text { Multivariable } \\
\text { AHR(95\% Cl)P-value }\end{array}$}} \\
\hline & & & & \\
\hline & $1.53(0.93-2.52)$ & 0.09 & & \\
\hline Age in year & $1.05(0.85-1.31)$ & 0.62 & & \\
\hline Disclosure: Not disclosed & $1.61(0.87-2.96)$ & 0.126 & & \\
\hline Functional status : Not workable & 5.54(3.37- 9.11) & $<0.001$ & $2.61(1.40-4.87)$ & 0.003 \\
\hline WHO clinical stage: Stage III and IV & $5.24(3.17-8.66)$ & $<0.001$ & & \\
\hline CD 4 cell count $:<=450$ copies $/ \mathrm{mm}^{3}$ & $5.74(1.79-18.3)$ & 0.003 & $3.81(1.17-12.39)$ & 0.026 \\
\hline Viral re-suppression: not re-suppressed & $11.2(6.7-18.6)$ & $<0.001$ & $6.87(3.86-12.23)$ & $<0.001$ \\
\hline Time to switch : Not timely switched & $3.43(1.69-6.94)$ & 0.001 & & \\
\hline
\end{tabular}

